\section{Signal transduction gets hairy}

Efforts to elucidate an important developmental signaling pathway have opened the door to a broad range of biotechnology applications-and generated an extremely hairy mouse. The mouse, which expresses a truncated, constitutively active form of the betacatenin signaling protein in its skin cells, generates new hair follicles as an adult. Ordinarily, new follicle formation stops before birth. While the discovery, reported in the November 25 issue of Cell (95:605), received extensive popular coverage as a potential cure for bald-

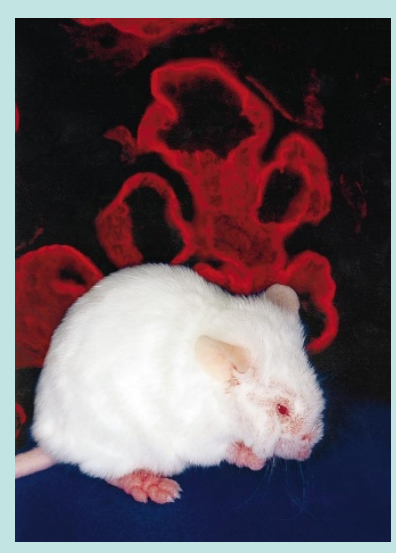

dependent upon whether animal skin could be induced to make hair follicles without progressing to skin tumors," explains Elaine Fuchs, a professor in the Howard Hughes Medical Institute at the University of Chicago and senior author on the study. While the tumors appear late in the animal's life and are relatively benign, they are potentially disfiguring. Fuchs suggests that a more imminent application might be the production of transgenic sheep which yield more wool. The team is currently trying to develop a more ness, the researchers emphasize that sub- detailed model of the signaling pathway, stantial barriers remain to such an applica- which may also be involved in the develoption. "Whether such technology would be ment of teeth, nails, mammary glands, and advantageous in a clinical setting would be other structures.

\section{RNA vaccine}

A new twist on genetic immunization has been reported by researchers at the University of Vienna, Austria. Until recently, most genetic vaccines under development have been based on DNA; however, in a recent report (Nat. Med. 4:1438, 1998), Christian Mandl and his colleagues have demonstrated the efficacy of an RNA-based vaccine. They showed that mice inoculated with an RNA vaccine developed from the virus that causes tick borne encephalitis (TBE) survive subsequent infection with wild-type TBE virus. After in vitro synthesis of TBE genomic RNA in Escherichia coli, the RNA was purified, used to coat gold beads, and inoculated into mice using a gene gun. According to the authors, only $0.1 \mathrm{ng}$ of RNA was sufficient for an infectious dose. In contrast, an attenuated form of the virus created by mutations in a noncoding region of the virus was able to confer protection against live virus at a dose of $5 \mathrm{ng}$ of RNA. In the future, vaccine developers will have to grapple with the thorny issue of whether DNA or RNA is the better vaccine candidate: "Using RNA, you eliminate some of the concern for potential DNA integration," says Margaret Liu, vice president of vaccine development at Chiron Corporation (Emeryville, $\mathrm{CA})$, "However, there is a possibility of reversion with RNA vaccines."

Research News Briefs written by Alan Dove, Margret Einarson, and Musa M. Mhlanga

\section{Silencing gene silence}

Plant biotechnologists have a love-hate relationship with the phenomenon known as posttranscriptional gene silencing (PTGS), a mechanism by which plant cells shut down the expression of highly abundant transcripts. PTGS appears to have evolved as a defense against viruses, raising the possibility that plants could be "immunized" with viral transgenes. At the same time, the phenomenon often prevents high-level expression of desirable transgenes. Now, researchers at Washington State University (Pullman, WA) report in the November 13 issue of Cell $(95: 461,1998)$ the discovery of a viral protein that shuts off PTGS. Cells expressing the P1/HC-Pro gene from tobacco etch virus permit high-level expression of a reporter gene that would ordinarily be silenced. While the viral gene would presumably facilitate the expression of transgenes in plants, James Carrington, a professor in the Institute of Biological Chemistry at Washington State and senior author on the study, warns that silencing is still a doubleedged sword: "A shutdown of gene silencing would likely enhance susceptibility to most viruses. This could potentially be a problem in large-scale outdoor field releases." Nonetheless, several companies are exploring the possibility of using $\mathrm{P} 1 / \mathrm{HC}$-Pro to suppress PTGS, and Carrington hopes that the finding will aid in uncovering the underlying mechanism of the phenomenon.

\section{Plant polyketides}

Using reverse genetics and biochemistry, a German-Finnish collaboration headed by Joachim Shroeder and Teemu Teeri have unmasked a role for a member of the chalcone synthase (CHS) family, gchs2 which suggests that CHS-related enzymes are involved in the biosynthesis of a much larger range of plant products than previously realized (Nature $396: 387,1998)$. The CHS superfamily synthesizes pyrones by using various substrates in a three-step condensation reaction of the polyketide pathway. Deploying an antisense version of a cDNA from a CHS-like protein from the ornamental plant Gerbera hybrida, the team was able to phenocopy a null mutation in gchs2. They then used nuclear magnetic resonance and mass spectroscopy to compare products of secondary metabolic pathways in wild type and "mutant" plants, identifying two compounds whose biosynthesis was not known to originate from the polyketide pathway. What's more, these compounds have known biological activities-they confer insect and pathogen resistance.

\section{Oocyte gene transfer}

Taking advantage of what may be an evolutionarily conserved "back door" to genomic modification, researchers at Gala Design (Sauk City, WI), the University of Wisconsin, Madison, and the University of California, San Diego have developed a technique that may have extensive applications in generating transgenic animals. During the MII meiotic arrest in oocyte development, the nuclear envelope breaks down. Reasoning that this event would allow access to the genome, the scientists infected bovine MII oocytes with a pseudotyped recombinant retrovirus carrying a reporter gene. The virus integrated efficiently, and, as the team reports in the November PNAS (95:14028, 1998), embryos derived from the infected oocytes carried the gene in all cells. Robert Bremel, managing director of Gala Design and senior author on the work, argues that the permeability of arrested oocytes to gene insertion may have played a role in mammalian evolution, allowing naturally occurring retroviruses or transposons to deliver useful traits: "Operating repeatedly through many generations, this system would provide a mechanism for periodic, dramatic quantum changes in phenotype." Bremel adds that the pseudotyped retrovirus allows researchers to take control of this process for a broad range of species, since "these vectors are virtually universal gene transfer entities." The company plans to focus initially on expressing novel proteins in transgenic cows' milk. 